\title{
DESARROLLO HUMANO EN ESPACIOS TURÍSTICOS
}

\author{
HUMAN DEVELOPMENT IN TOURISM SPACES
}

\author{
Ana María Huaita Alfaro \\ University College London, Reino Unido
}

\section{RESUMEN}

La importancia ganada por el sector turismo en la actualidad invita a analizar su estrecha relación con el desarrollo humano y así complementar la clásica visión de desarrollo económico aplicada a la evaluación de este sector. Para ello se introduce el enfoque de desarrollo humano como expansión de capacidades, con el propósito de observar cómo es posible a partir del turismo atender de manera integral las necesidades de bienestar de las personas. Aplicando este enfoque de capacidades se analizan los procesos y elementos que definen el contexto turístico, y se identifican oportunidades y riesgos a los cuales se debe hacer frente para alcanzar experiencias enriquecedoras en él. En particular, se resalta la importancia de observar los encuentros entre locales y visitantes, y las interacciones en torno a la cultura como dinámicas facilitadoras para un desarrollo inclusivo y sostenible.

Palabras clave: Cultura, desarrollo humano, enfoque de capacidades, gestión del desarrollo, inclusión, participación, Perú, turismo.

\begin{abstract}
The current importance achieved by the tourism sector leads to the analysis of its close relationship with human development, thus complementing the classic vision of economic development applied when evaluating this sector. In this intention, the human development and capabilities approach is introduced in order to observe how it is possible to broadly meet needs in terms of human well-being from tourism. Processes and elements defining the tourism context are analysed applying the capabilities approach, and opportunities and risks to achieve enriching experiences within this context are identified. In particular, it is highlighted the importance to observe encounters between locals and visitors as well as interactions around culture as dynamics facilitating an inclusive and sustainable development.
\end{abstract}

Key words: Culture, human development, capabilities approach, development management, inclusion, participation, Peru, tourism 


\section{Introducción}

Se considera al turismo como una actividad económica que tiene la potencialidad de contribuir sustancialmente a la disminución de la pobreza, especialmente cuando se desarrolla con la participación directa de las poblaciones involucradas (Huaita, 2012). Por ello, muchos proyectos de desarrollo han sido formulados en esta línea, dando lugar a oportunidades laborales y al incremento de ingresos a partir de la provisión de servicios turísticos, principalmente asociados al disfrute de atracciones naturales y culturales de las localidades (Telfer, 2003). Sin embargo, es necesario ampliar la concepción que se tiene del turismo y hacer explícita su relación con el desarrollo humano trascendiendo la dimensión económica, observando cómo es posible a partir de este atender de manera integral las necesidades de bienestar de las personas.

Para efectos de este análisis se propone observar al turismo no como una actividad o como un sistema de elementos fijos, sino como un campo donde la confluencia de diversos procesos sociales y la interacción con la cultura ofrecen oportunidades para alcanzar avances sostenibles en el bienestar de las personas (Alkire \& Deneulin, 2009; Sen, 1999; UNDP, 1990). En consecuencia, se propone ahondar en el análisis del turismo desde un enfoque de desarrollo humano, donde se resalte la centralidad de la expansión de capacidades y libertades humanas a partir de las experiencias turísticas. La disponibilidad de oportunidades para el logro de condiciones de bienestar desde estas experiencias es altamente dependiente de las particularidades del contexto institucional y cultural donde se desenvuelven los individuos y es por ello que el análisis y práctica del turismo deben emplear lentes que abarquen los múltiples ámbitos que este implica (Rodríguez, 2008).

\section{El camino hacia el desarrollo humano}

Según la coyuntura y las corrientes de pensamiento reinantes, el desarrollo ha sido entendido desde perspectivas que divergen en sus fundamentos pero convergen, al menos en la teoría, en asumir que el objetivo final del desarrollo está en la mejora del bienestar de las personas. Es ello lo que lo convierte en un tema de revisión permanente y también en una preocupación latente para todo aquel que considere influir desde su accionar en su destino individual y colectivo.

En la práctica, el bienestar se ha pretendido entender como una situación a nivel macro y se le ha relacionado en primer lugar con la eliminación de la pobreza y con la bonanza económica. Por ello el desarrollo ha estado ampliamente ligado a enfoques de crecimiento económico como la base para el logro de este bienestar. Estos enfoques se han centrado principalmente en observar el incremento de ingresos y rentas como sinónimos de mejoras en la calidad de vida, imponiendo así una visión ciertamente reduccionista de lo que esta última implica (Spence, 2009). Más aún, el crecimiento económico ha tomado tradicionalmente las orientaciones impuestas por aquellos actores de mayor poder político y económico, no respondiendo de esta manera a las necesidades de las mayorías en condiciones menos favorables (North, 1990).

A pesar de que esta perspectiva aún define muchas de las políticas y acciones en la gestión del desarrollo, hay una creciente atención por lograr un desarrollo más integral. La pobreza y las limitaciones al bienestar pueden experimentarse en múltiples dimensiones y estas no recaen únicamente en la satisfacción de necesidades 
económicas. Esto lleva a poner énfasis en las necesidades de las personas y a tomar en cuenta las diferentes dimensiones -como las referentes a procesos políticos, sociales y medioambientales- sobre las que estas limitaciones recaen.

Las diversas crisis financieras y los conflictos sociales, el desigual crecimiento interno y la falta de equidad en oportunidades, entre otros retos para la gestión del desarrollo en la actualidad, han puesto en evidencia las demandas por renovar las perspectivas frente a la comprensión del desarrollo y el bienestar. Estas situaciones han llevado a revisar procesos como la distribución de capitales, las instituciones, las particularidades de los contextos sociales y la capacidad de los sistemas de gobierno para satisfacer las demandas de calidad de vida y sostenibilidad por parte de las poblaciones (Donelly, 1999). De esta forma, renovados enfoques basados en el desarrollo a nivel de las personas como base para logros a nivel macro han ido ganando espacios en una nueva agenda para el desarrollo a escala internacional (Arbour, 2006).

\section{Un paradigma de desarrollo basado en las capacidades}

Un paradigma central para el desarrollo humano es aquel identificado como el enfoque de capacidades o capability approach. Desde este enfoque, el desarrollo humano se entiende como un proceso de expansión de libertades individuales al cual se llega a través de la "expansión de capacidades de las personas para ejercer el tipo de vida que valoran" (Sen, 1999: 18).

De acuerdo a Sen (1999), la relevancia de las libertades recae en que ellas cumplen dos roles fundamentales en el proceso de desarrollo. Las libertades humanas son en sí mismas metas del desarrollo, puesto que su logro implica la superación de una serie de privaciones en el bienestar de las personas. A su vez, las libertades son medios para el desarrollo dada su función como instrumentos para la superación de dichas privaciones. Así, las capacidades son entendidas como las posibilidades de tener y hacer aquello que una persona valora en las diversas dimensiones de la vida, apuntando a logros que trascienden aquellos en términos materiales (Sen, 1992, en Samman \& Santos, 2009).

\section{Perspectivas sobre la promoción del desarrollo}

Al entender el desarrollo humano como un proceso de expansión de capacidades individuales es posible identificar dos subprocesos clave por observarse desde la teoría y la práctica (UNDP, 1990):

1. La formación de las capacidades (por ejemplo, a través de condiciones como servicios mejorados de educación y salud, y recursos para el enriquecimiento de conocimientos y habilidades).

2. El uso que las personas hacen de las capacidades adquiridas (por ejemplo, a través de su participación en actividades productivas, actividades de ocio o en asuntos políticos, culturales o de decisión pública).

Ambos subprocesos deben ser considerados en la gestión del desarrollo y llaman a dedicar una especial atención a la ampliación de oportunidades que permitan a las personas concretarlos. Ante la complejidad de este reto, se resalta la importancia del involucramiento de instituciones sociales, políticas, económicas, entre 
otras -formales e informales-, que incidan en el ámbito local pero también que lo trasciendan. Estas son, por ejemplo, "el Estado, el mercado, el sistema legal, los partidos políticos, los medios de comunicación, los grupos de interés público y los foros de discusión pública, entre otros” (Sen, 1999: xii-xiii, traducción libre).

Las instituciones definen en gran medida la formación y uso de capacidades a partir del establecimiento de condiciones que facilitan la eliminación de privaciones a libertades básicas tales como: limitadas opciones para la generación de ingresos, pobreza, inequidad social sistemática, intolerancia, estados represivos, malnutrición, mortalidad prematura, entre otras (Johnson, 2009; Sen, 1999). Por ejemplo, los sistemas democráticos permiten una participación política abierta y generan espacios públicos donde todos pueden expresar sus aspiraciones y creencias. Más aún, brindan condiciones para prevenir la centralización de poderes. Asimismo, reglas justas para el acceso a mercados pueden impulsar la productividad y facilitar una distribución equitativa de bienes entre la población (Evans, 2004).

En general, este enfoque pone en primer plano las necesidades y aspiraciones reales de las personas, y sobre todo la ampliación de sus capacidades para que puedan actuar como agentes en la construcción de sus destinos y los de sus comunidades (Samman \& Santos, 2009; Sen, 1999). Además, se realza la centralidad de reconocer las particularidades de cada contexto y de fortalecer las instituciones sociales y la autodeterminación de los individuos para promover procesos participativos que aseguren acciones efectivas, en términos del logro de dichas necesidades y aspiraciones (Chambers, 1997; Chopra \& Duraiappah, 2008; Deneulin, 2008; Putnam, 1993; Sen, 1999). Se puede afirmar entonces que los elementos fundamentales de este enfoque son la agencia (entendida como auto-eficacia de los individuos), las relaciones de poder (entre los diversos actores que interactúan en el proceso de desarrollo) y participación (de todos estos actores, de manera inclusiva) (Frediani, 2010).

Estas consideraciones llevan a identificar la importancia de evaluar los mecanismos o procesos que conducen al desarrollo y, dentro de ello, la relevancia de observar las relaciones entre las personas y su contexto (Chopra \& Duraiappah, 2008; Dreze \& Sen, 2002). La promoción del desarrollo, en la teoría y en la práctica -desde el estudio de la realidad hasta la gestión e implementación de proyectos-, puede abordarse a partir de los múltiples campos donde se desenvuelven las dinámicas humanas. En cada uno de ellos son identificables los aspectos antes resaltados para poner en marcha un proceso de desarrollo centrado en las personas.

A manera de ilustración y respondiendo al interés de nuestro estudio, se observará al turismo como uno de estos campos desde donde puede evaluarse el desarrollo sobre la base del enfoque de capacidades.

\section{Las personas primero: reflexiones sobre los estudios y gestión del desarrollo turístico}

El turismo ha sido largamente descrito como un negocio y como un fenómeno social, resaltando la marcada importancia que se otorga a la generación de capitales a partir de él. Del mismo modo, se le ha observado como un "set predominantemente exótico de productos de consumo que ocurren en lugares y momentos específicos" (Larsen, Urry \& Axhausen, 2007: 245). Se le ha descrito también como un sistema, restándole 
características propias de su dinamismo e identificando en él elementos fijos como son "demanda de viaje, intermediarios turísticos, características del destino y consecuencias" (Nogués, 2008: 144).

Estas concepciones, coincidentes en buen grado, han orientado tanto los programas para el desarrollo turístico como los estudios realizados sobre el tema. Así, se han generado discursos acerca de sus diversos impactos otorgándoles una connotación de peligrosos para los destinos turísticos. También se han evaluado las tendencias y experiencias de consumo por parte de los visitantes, privilegiando la generación de ingresos y gestionando las actividades turísticas de manera ajena de las comunidades; es decir, sin considerar a los lugareños en la planificación del desarrollo turístico (Flacke-Neudorfer, 2008). Sobre este último punto hay numerosos ejemplos donde la bonanza económica generada por el turismo no se refleja en el bienestar de la población del destino en cuestión.

Es así que se ha dejado de lado la adopción de perspectivas que permitan observar al turismo dentro de un marco más amplio, en el cual pueda verse de manera más explícita su relación con el desarrollo. Ante estas consideraciones, se propone identificar al turismo como un campo o un contexto en sí mismo, el cual se rige por las dinámicas de los actores y otros componentes que alberga -como instituciones, recursos y procesosy donde todos cumplen un rol importante en atender a las necesidades reales de las personas.

En esta dirección han surgido interesantes enfoques para el estudio y desarrollo del turismo. Por ejemplo, Nogués (2008: 145) sugiere que es central entender cómo las relaciones entre individuos y sus actividades en un contexto turístico otorgan significados y sentidos particulares a "nociones como sostenibilidad, desarrollo, turismo y patrimonio cultural". Por otro lado, Flacke-Neudorfer (2008: 246) resalta la importancia de entender "lo que las personas hacen con y dentro del turismo" y llama a dejar atrás el clásico enfoque (cuantitativo) de seguimiento de sus impactos.

El turismo se asimila dentro de las dinámicas de la posmodernidad, como la globalización, la creciente facilidad para la movilidad espacial - con motivo de actividades como migración, trabajo, estudio, vida familiar y amical, etc.-, la predominancia de las comunicaciones virtuales, la apertura a la socialización entre agentes de diversas procedencias, entre otras. A través del turismo se han incrementado los momentos de encuentro entre visitantes y locales, generándose cambios en la manera de experimentar los desplazamientos y los encuentros (Urry, 2002a). De esta forma, las interacciones entre personas permiten a los individuos tomar conciencia de su pertenencia a un mundo social mucho más amplio que se despliega ante ellos (Melville, Selby

\& Cliff, 2007). Asimismo, las interacciones con el entorno influyen en la percepción de los componentes del mundo natural y construido que forma parte de las experiencias turísticas.

Estas observaciones permiten reconocer en el turismo su estrecha relación con el desarrollo de las personas, especialmente cuando se incluye a las poblaciones de los destinos turísticos desde la concepción de los proyectos y actividades. Como señala Santana (2003: 10), "el turismo ya no es algo externo a las sociedades" y, por consiguiente, no es externo a los individuos que las componen, participen o no directamente de la oferta/demanda de productos turísticos. 
De esta forma se evidencia el hecho de que el turismo se hace más efectivo para el desarrollo humano cuando se gestiona con un enfoque desde el interior de las comunidades, pero también se deja entrever que el progreso del turismo recae ampliamente en la forma en que es asimilado por las personas en la actualidad. Esto último está íntimamente relacionado con las manifestaciones culturales que confluyen en la experiencia turística y es por ello que en este punto es importante observar más de cerca el rol de la cultura.

\section{Cultura y turismo}

Dadas las ideas expuestas previamente, es importante mencionar el lugar central que se ha otorgado a la cultura en los discursos y políticas para el desarrollo del turismo. En concordancia con el enfoque de capacidades, la libertad para tomar parte de manifestaciones culturales es básica para el desarrollo humano y, a partir de ella, se satisfacen necesidades y se alcanzan otras libertades fundamentales para los individuos (Sen, 2004).

La cultura puede entenderse como "aquella mezcla de manifestaciones, modos, lo que se dice, lo que se hace, circunstancias y contextos que adquieren su sentido dentro de un grupo específico y dan sentido a la vida social..." (Nogués, 2008: 145, traducción libre). Esto incluye dentro de la cultura a los valores sociales, las normas, las tradiciones y otros aspectos que se (re)crean a partir de la convivencia en un colectivo y localidad, y le dan sentido a la vida en su interior. A su vez, dentro de un mismo colectivo pueden convivir diversas culturas provenientes de las diferentes afiliaciones y bagajes de los individuos que lo integran, y sobre los cuales forman sus identidades. Los intercambios entre culturas diversas hacen que se compartan recursos y que estas experimenten constantes evoluciones (Sen, 2004). Esto permite identificar el valor de las contribuciones de las diferentes culturas al enriquecimiento continuo de las personas y, con ello, la importancia de la apertura a la diversidad cultural.

No obstante, el rol de la cultura frente a la intersección del turismo y desarrollo es con frecuencia reducido a ser únicamente un medio para el desarrollo económico y para el éxito de las prácticas turísticas. En particular, el patrimonio cultural es identificado para estos efectos. El patrimonio puede entenderse como el bagaje cultural e histórico que surge a partir de las experiencias vividas por individuos de otros tiempos. Este patrimonio adquiere contemporaneidad a través de los individuos que lo comparten de diferentes maneras en la actualidad, incluso mediante las prácticas turísticas, pues estas "le dan vida y lo transforman en actos de percepción y conocimiento de una época, de un lugar o de una experiencia...” (Urbano, 2012: 189). Más aún, el patrimonio cultural se introduce en la vida contemporánea a través de la oferta, demanda y consumo de productos y servicios que forman parte de las experiencias a partir del turismo, involucrando así a visitantes y locales (Urbano, 2012).

Por tanto el patrimonio, así como otros aspectos culturales, tiene un valor mucho mayor al normalmente observado. El enriquecimiento de la vida a través de la cultura permite a las personas observarse de manera más plena en su contexto: desarrollando habilidades sociales, mejorando su participación en sociedad, valorando y apropiándose de recursos locales, así como de los provistos por otras culturas. Del mismo modo, este enriquecimiento se traduce en el planteamiento de aspiraciones, toma de decisiones y uso de oportunidades del contexto para concretar mejores opciones para su futuro (Appadurai, 2004; Robinson \& Picard, 2006). 
Por ello resultan contraproducentes las visiones que limitan la función de la cultura para el florecimiento humano; por el contrario, debe observarse cómo "las propuestas de desarrollo encuentran múltiples posibilidades de articulación con la cultura” (Rey, 2009) y sentar las condiciones para que estas puedan ser puestas en práctica.

En este sentido, existen aspectos que permiten vislumbrar potencialidades a partir de las experiencias culturales y turísticas para el desarrollo humano. Por ejemplo, el sector cultural ha sido identificado como un importante generador de empleos, promotor de innovaciones tecnológicas y facilitador de transacciones económicas a nivel global. La escala de movilización de recursos y beneficios desde este sector llama a fortalecer la participación de los gobiernos en el acompañamiento de estos procesos. A la vez, se hace evidente la necesidad de un mayor involucramiento de agentes del sector privado y de la sociedad civil con el propósito de facilitar un desarrollo sostenido, así como equidad e inclusión en el mismo. Esto debe verse traducido en la concepción de lugares, ciudades y pueblos convertidos en espacios -sociales y físicos- que propicien encuentros para promover la creatividad, el planteamiento de aspiraciones y los emprendimientos de las personas, incitadas por su paso a través de estos espacios.

\section{Turismo y desarrollo para todos}

Para que el campo turístico se convierta verdaderamente en uno donde pueda accederse a logros en términos de desarrollo humano, es importante identificar factores centrales, dependientes de cada contexto, que condicionen que esta relación turismo/desarrollo humano se concrete efectivamente. En este sentido, se puede mencionar factores claves y generales al contexto de nuestro país, como la mejora y la ampliación del acceso a la educación formal, la aceptación de la diversidad cultural - tanto al interior de las comunidades de los destinos turísticos como de los visitantes- y el acceso a recursos que permitan el desarrollo de actividades productivas (medios de financiamiento, conocimiento pertinente a dichas actividades y tecnología). Es decir, es necesario un contexto social e institucional que sustente la apertura al turismo y facilite el desarrollo de capacidades para la creación de soluciones innovadoras de promoción del sector y, a través de ello, la mejora de la calidad de vida (por ejemplo, mediante el establecimiento de negocios locales).

Estas observaciones permiten identificar a la vez la importancia de la formación de "públicos" o consumidores, y de "creadores" o emprendedores, con el fin de sentar las bases para una interacción positiva entre los agentes involucrados en el desarrollo turístico y entre ellos y el destino de interés (Aguilar, Hiernaux

\& Lindón, 2006; Appadurai, 2004; Florida, 2003). Así se incrementa el involucramiento y acceso a oportunidades a partir de un campo en crecimiento como el turístico, y se aprovechan de manera más amplia las opciones que ofrece la modernidad (Urry, 2002a; Urry, 2002b). De esta forma, puede observarse cómo el crecimiento del turismo en una localidad y el bienestar de su sociedad están muy relacionados con el buen funcionamiento de las instituciones que inciden en ella.

Un lugar crece en atractivo turístico junto con el incremento de oportunidades que ofrece para que las personas puedan disfrutar de libertades que les permitan tener logros de vida que valoran (Urry, 2002a). El turismo puede identificarse como un campo donde tanto visitantes como locales encuentran recursos para su 
propio desarrollo a partir del disfrute de actividades e interacciones diversas (en buen grado en torno a expresiones culturales). Estas experiencias permiten a su vez que las personas accedan a las potencialidades que ofrece el mundo moderno al reducir cada vez más los límites para transcurrir entre lugares y momentos, y con ello aprovechar al máximo las capacidades que gana al interactuar con sociedades y sus creaciones (Melville et al., 2007; Urry, 2002a).

\section{Los retos}

El disfrute de actividades culturales y turísticas, tanto por parte de los visitantes como de los locales, implica una apertura a la diversidad cultural y la tolerancia hacia personas "externas" a las comunidades receptoras, al igual que una capacidad adecuada para atender la demanda de productos y servicios por los visitantes. Sin embargo, con frecuencia se han alimentado discursos de protección de lo local y se han resaltado los riesgos antes que los beneficios que conllevan las experiencias turísticas y la interacción entre individuos de diferentes culturas. Así, se ha llegado a promover un cierto extremismo en el desarrollo de lazos entre los miembros de una comunidad, basándose en la búsqueda de conservación del patrimonio y de la tradición compartidos. Esto puede ser perjudicial en el sentido que lleva a la exclusión de individuos externos a la comunidad y al rechazo de su cultura, y con ello a inherentes limitaciones en oportunidades para su crecimiento.

No obstante, es importante hacer prevalecer la libertad de los locales para decidir la apertura o no al desarrollo de actividades turísticas y al consumo del patrimonio cultural que albergan. También es importante poner en valor la participación y el trabajo de los locales, y no permitir que el turismo beneficie únicamente a aquellos con mayor poder político y económico. Es común observar el poco valor que se otorga a la formación de las poblaciones en los destinos turísticos con el fin de facilitar su participación en este campo. Esto se refleja en múltiples aspectos, como los deficientes (o ausentes) servicios educativos y de capacitación para el trabajo, así como la explotación de la mano de obra (bajos salarios, horarios extensos, pésimas condiciones para laborar, entre otros aspectos).

Todo ello impide que las personas encuentren verdaderamente en el campo turístico medios para la mejora de su calidad de vida. El rechazo a esta realidad puede verse reflejado en conflictos varios, como los que se producen al interior de las comunidades - por ejemplo, cuando hay intereses contrarios, lo cual podría llevar al fraccionamiento de estas- o entre las comunidades, los consumidores y los promotores de turismo (agentes del sector público y privado). También puede ocasionar que los individuos de las localidades tomen decisiones que van en detrimento de su desarrollo, con la intención de obtener mejores condiciones de trabajo y mejores salarios - por ejemplo, el abandono de la escuela con el propósito de buscar mejores ingresos para la familia o el escape del hogar/localidad también por aspiraciones de mejores ingresos--, enfrentando sentimientos de desarraigo y las duras condiciones que implican el empezar desde cero.

De otro lado, los discursos en defensa de lo local han asumido al bagaje histórico y cultural como un conjunto de normas o pautas que definen de antemano la forma en que los individuos ingresan a la sociedad, negando 
de esta forma su libertad de construir su propia identidad. Esto último es particularmente relevante, puesto que la construcción de la identidad implica el uso de la razón y de las capacidades que las personas adquieren durante la socialización, así como la asimilación de las múltiples afiliaciones que les ofrece el mundo moderno (Sen, 2006). Si disponen de las libertades para que ello ocurra, las personas pueden intervenir en el establecimiento de espacios receptivos a la diversidad y, a la vez, facilitadores de experiencias turísticas enriquecedoras. Se cumple así que "las culturas vivas son tan sólidas que pueden asumir la globalización [entre otros procesos contemporáneos] sin que esta sea traumática" (Hulerig, 2006) y sin restar los beneficios para el florecimiento humano antes resaltados para el turismo.

\section{Las oportunidades}

El turismo tiene la potencialidad de contribuir al desarrollo desde múltiples dimensiones. Por el lado de la cultura, puede sustentar la construcción de valores como la solidaridad y el respeto por las diferencias culturales o de credos. Esto se ve traducido en un tejido social fortalecido, donde las tensiones sociales se ven disminuidas. Más aún, puede contribuir a incrementar la participación de los habitantes en la preservación de las tradiciones y del patrimonio que comparten, identificándolos como activos que enriquecen su experiencia de vida en su localidad, así como la experiencia de quienes la visitan.

Incluso los lugares de procedencia de los visitantes son influenciados por la vivencia turística, ya que participan del intercambio de recursos culturales (valores, costumbres, expresiones), financieros (a través de los gastos que implica la visita), entre otros (Urry, 2002a). Por otro lado, la apertura a un campo como el turismo permite el incremento de oportunidades laborales y la diversificación de mecanismos para la generación de ingresos.

Así, sobre la base de un tejido social fortalecido y de las capacidades desarrolladas en las dinámicas turísticas, cabe promover acciones conducentes al desarrollo de manera sostenida. Por ejemplo, se potencia el desempeño de las personas en diferentes espacios y procesos sociales que las afectan y eso cala en su participación cívica y política. Del mismo modo se da lugar a relaciones cooperativas en las actividades económicas, donde se promueve la participación del sector privado. Con ello se da paso a una nueva forma de entender el neoliberalismo, un "neoliberalismo social" (Andolina, Laurie \& Radcliffe, 2009), el cual también promueve el desarrollo de capacidades a partir de la experiencia de interactuar con agentes de este sector y la participación en mercados.

Estas observaciones pueden trasladarse al caso particular de Perú, donde el turismo, tanto interno como receptivo, ha tenido un crecimiento sustancial en los últimos años (Observatorio Turístico del Perú, 2013). Ello invita a plantear mecanismos que eliminen las profundas privaciones que experimentan numerosas comunidades del país y así permitir a la población lograr mayores beneficios a partir de estas oportunidades. La capacitación e inclusión de los pobladores en líneas estratégicas para el crecimiento del país, como es el caso de la promoción del turismo, son grandes tareas aún por emprender. Para ello debe aplicarse una perspectiva basada en las personas y atenderse cuestiones centrales como: ¿qué es lo que esperan las 
personas en su localidad a partir del turismo?, ¿de qué manera conciben ellas su participación en las actividades turísticas?, ¿cómo se refleja su participación en logros de calidad de vida?, ¿qué significados y valores renovados se otorga a los elementos del contexto turístico?, ¿cómo esto da nuevos sentidos a la vida social en la localidad?, entre otras.

\section{Caso de estudio: "Porteadores" del Camino Inca (Cuzco, Perú)}

Una reciente investigación enfocada en las comunidades rurales cercanas a la ruta turística conocida como Camino Inca, que conecta la ciudad de Cuzco con la ciudadela de Machu Picchu, permite observar varias implicancias del turismo en términos de desarrollo humano para los miembros de dichas comunidades. De manera central, la investigación aborda las experiencias de los pobladores que trabajan como "porteadores" (cargadores y proveedores de servicios a turistas durante su recorrido por el Camino Inca) y los cambios que reportan en sus aspiraciones y acciones para lograr una mejor calidad de vida. Las comunidades en las cercanías del Camino Inca, al igual que otras numerosas comunidades situadas en la zona sur de los Andes de Perú, han observado el crecimiento de ciudades cercanas pero han permanecido ajenas a los beneficios derivados de estos avances. Incluso a pesar del incremento del flujo turístico, como ha sido el caso del que se produce hacia Machu Picchu dado su creciente reconocimiento internacional, las intervenciones por parte de los sectores público y privado han estado dirigidas a atender directamente la demanda turística y asegurar la generación de ingresos a partir de las actividades de este sector, sin un enfoque de sostenibilidad social.

Ante esta situación, los testimonios de los pobladores de las zonas estudiadas reflejan las profundas limitaciones que ellos perciben, no solo por la situación económica de sus familias sino también por el propio contexto. Elementos clave identificados en dicho contexto han sido, por un lado, aquellos relacionados a lo impuesto por instituciones externas a las comunidades: insuficiente acceso a servicios de educación y salud, pobres condiciones de vivienda y nutrición, prevalencia de medios de trabajo de alta demanda física y mínima retribución económica, ausencia de actores e instituciones que puedan generar un cambio a las duras y restrictivas realidades en las que se desenvuelven (como ejemplo, la falta de mecanismos para hacer cumplir una ley que establece condiciones dignas de trabajo para los "porteadores"). Por otro lado, los "porteadores" identifican elementos que derivan de las instituciones y normas locales, como las débiles relaciones entre algunos miembros de la comunidad e incluso al interior de las familias (reportando en estas últimas casos de violencia), así como la poca eficiencia de las organizaciones locales para manejar las presiones de las actividades turísticas en las localidades.

Dentro de este contexto, los pobladores participantes del estudio han señalado al turismo como un sector que les ha permitido, a pesar de las duras circunstancias, encontrar nuevas formas de trabajo y nuevas formas de mirarse a sí mismos e identificar aspectos en los que desean progresar. Si bien los ha motivado a mejorar en términos de servicios y productos para satisfacer la demanda de los visitantes, los "porteadores" han identificado que el desarrollo de habilidades y la adquisición de conocimientos, a 
través de servicios educativos y de capacitación, son necesidades centrales para salir de la pobreza. La identificación de estos factores para su propio desarrollo ha surgido en buen grado a partir de las interacciones mantenidas con los turistas, a través del uso de habilidades de comunicación y el acceso a conocimientos varios, incluyendo un buen manejo en más de un idioma. Sin embargo, también se han observado conflictos de identidad entre los pobladores, pues las condiciones sociales en las que se desenvuelven llegan a ser más restrictivas que facilitadoras para el logro de sus aspiraciones. A pesar de ello, aquellos que han podido sortear dificultades y alcanzar logros de vida que valoran, han resaltado el rol central de las capacidades adquiridas para este propósito. Así, el caso de los "porteadores" permite observar cómo a través del turismo se presentan posibilidades para un replanteamiento de modos de vida individual y en comunidad, y hace evidente la disponibilidad o no de herramientas para redefinir un entorno físico y social de pobreza mantenido por generaciones.

Referencias: Arellano, Espinoza y Howitt, 2013; Rollefson, Espinoza y Arellano, 2012.

\section{A manera de conclusión}

A lo largo de estas reflexiones se ha buscado mostrar cómo el turismo tiene la potencialidad de generar espacios en los que las personas alcancen vidas individuales y sociales más fructíferas y plenas. Con este propósito, se ha identificado la relevancia de observar los procesos que ocurren dentro de las experiencias turísticas para determinar oportunidades y riesgos en términos de desarrollo humano, a los cuales se debe hacer frente para así enriquecer las experiencias en el contexto turístico. En particular, se ha valorado la importancia de los encuentros entre locales y visitantes, y el intercambio de experiencias en torno a la cultura como oportunidades para el desarrollo de capacidades.

Del mismo modo, centrar la evaluación del turismo y del desarrollo en los protagonistas -locales y visitantes- de dichas experiencias permite reconocer las diversas dimensiones donde las personas experimentan privaciones a sus libertades y donde centran sus aspiraciones. De allí la importancia de recoger esta información de los mismos actores involucrados, de reconocer su rol crucial en el desarrollo turístico y de priorizar mejoras en su bienestar antes que el beneficio económico de las minorías con mayor poder. De esta manera la gestión del desarrollo se vuelve más eficiente pues se identifican los campos y acciones donde debe trabajarse en forma prioritaria para atender libertades y aspiraciones, como la educación, la gestión territorial, la protección del patrimonio cultural, etc.

En la gestión del desarrollo, en general, la búsqueda de concertación entre el crecimiento económico con el progreso en otras esferas del desarrollo humano, de manera inclusiva y sostenible, presenta retos ineludibles para los agentes de gobierno, pero llama también a un mayor involucramiento desde otros sectores. Por ejemplo, un ámbito aún por explotar es el de la incorporación de actores del sector privado en la promoción de actividades que generen mayor participación de las poblaciones. 
Así, el enfoque de capacidades antes planteado resulta relevante en cuanto invita a colocar especial atención en la interacción entre las personas y entre estas y su contexto. Del mismo modo, permite analizar al turismo como un campo donde las personas interactúan con lugares y momentos donde nada es estático, ni las identidades, ni las relaciones ni los recursos presentes, pero donde el enriquecimiento y la evolución de las personas son permanentes.

\section{Referencias}

Aguilar, M., Hiernaux, D. \& Lindón, A. (2006). Lugares e imaginarios en la Metrópolis. Barcelona: Anthropos.

Alkire, S. \& Deneulin, S. (2009). A Normative Framework for Development. En S. Deneulin \& L. Shahani (Eds.). An introduction to the Human Development and Capability Approach. London: Earthscan.

Andolina, R., Laurie, N. \& Radcliffe, S. (2009). Indigenous Development in the Andes. Culture, Power, and Transnationalism. London: Duke University Press.

Appadurai, A. (2004). The Capacity to Aspire: Culture and the Terms of Recognition. En V. Rae \& M. Walton (Eds.). Culture and Public Action. Stanford: Stanford University Press.

Arbour, L. (2006). Foreword. En B. Andreassen \& S. Marks (Eds.). Development as a Human Right. Legal, Political, and Human Dimensions. London: Harvard University Press.

Arellano, A., Espinoza, C. \& Howitt J. (2013). I don’t want them to suffer like I have: Porters of the Inca Trail's views on education and aspirations for social change. En M. Gravari, L. Bourdeau \& M. Robinson (Eds.). World Heritage Sites and Tourism: Global and Local Relations. UK: Ashgate Publishing Limited.

Chambers, R. (1997). Whose Reality Counts? Putting the First Last. London: IT Press.

Chopra, K. \& Duraiappah, A. (2008). Operationalizing capabilities in a segmented society: the role of institutions. En F. Comim, M. Qizilbash \& S. Alkire (Eds.). The Capability Approach. Concepts, Measures and Applications. Cambridge: Cambridge University Press.

Deneulin, S. (2008). Beyond individual freedom and agency: structures of living together in the capability approach. En F. Comim, M. Qizilbash \& S. Alkire (Eds.). The Capability Approach. Concepts, Measures and Applications. Cambridge: Cambridge University Press.

Deneulin, S. \& Shahani, L. (Eds.) (2009). An introduction to the Human Development and Capability Approach. London: Earthscan.

Dreze, J. \& Sen, A. (2002). India: Development and Participation. New York: Oxford University Press.

Donelly, J. (1999). Human Rights, Democracy, and Development. Human Rights Quarterly, 21(3), 608-632.

Evans, P. (2004). Development as Institutional Change: The Pitfalls of Monocropping and the Potentials of Deliberation. Studies in Comparative International Development, 38(4), 30-52.

Flacke-Neudorfer, C. (2008). Actors or victims? Actor-oriented perspectives on new forms of tourism. Chapter 14. En P. Burns \& M. Novelli (Eds.). Tourism Development. Growth, Myths and Inequalities. Wallingford: CAB International.

Florida, R. (2003). Cities and the Creative Class. City \& Community, 2(1), 3-19.

Frediani, A. (2010). Sen's Capability Approach as a framework to the practice of development. Development in Practice, 20(2), 173187.

Huaita, A. M. (2012). Ecoturismo y desarrollo humano: el caso de una asociación privada con la Comunidad de Infierno. Turismo y Patrimonio, 7, 23-31.

Hulerig, E. (2006). El retorno de la identidad étnica: Entrevista a Luis Millones. Gaceta Cultural del Perú, 24, 14-15.

Johnson, S. (2009). Institutions, Markets and Economic Development. En S. Deneulin \& L. Shahani (Eds.). An introduction to the Human Development and Capability Approach. London: Earthscan. 
Larsen, J., Urry, J. \& Axhausen, K. (2007). Networks and Tourism. Mobile Social Life. Annals of Tourism Research, 34(1), $244-262$.

Melville, R., Selby, M. \& Cliff, M. (2007). Re-telling the city. Exploring narratives of Liverpool. Impacts 08. The Liverpool Model, European Capital of Culture Research Programme.

Nogués, A. M. (2008). A contextual approach to the power relation between tourism and development (Chapter 10). En P. Burns \& M. Novelli (Eds.). Tourism Development. Growth, Myths and Inequalities. Wallingford: CAB International.

North, D. (1990). Institutions, institutional change and economic performance. Cambridge: Cambridge University Press.

Observatorio Turístico del Perú, BADATUR. Perú: Crecimiento del turismo receptor. (Último acceso: 01/04/2013). Recuperado de www.observatorioturisticodelperu.com/mapas/ctrtotal.pdf

Putnam, R. (1993). The prosperous community: social capital and public life. American Prospect, 13, 35-42.

Rey, G. (2009). Industrias culturales, creatividad y desarrollo. Madrid: Agencia Española de Cooperación Internacional para el Desarrollo.

Robinson, M. \& Picard, D. (2006). Tourism, Culture and Sustainable Development. Programme "Culture, tourism, development". Division of cultural policies and intercultural dialogue, Culture Sector, UNESCO. Paris: UNESCO.

Rodríguez, A. (2008). Tourism, Indigenous People and Conservation in the Ecuadorian Amazon. En A. Stronza \& W. Durham (Eds.). Ecotourism and Conservation in the Americas. Trowbridge: Cromwell Press.

Rollefson, J., Espinoza, C. \& Arellano, A. (2012). Responsible Tourism and Poverty: The Porters of the Inca Trail. En Coloquio International Rutas Turísticas e Itinerarios Culturales: entre la memoria y el desarrollo. Université Laval (Québec, Canadá) 13 al 15 de junio de 2012. Actas del Coloquio, Volumen II, 492-499.

Samman, E. \& Santos, M. (2009). Agency and Empowerment. A review of concepts, indicators and empirical evidence. Draft paper prepared for the 2009 Human Development Report in Latin America and the Caribbean. Oxford Poverty and Human Development Initiative, University of Oxford.

Santana, A. (2003). Patrimonios culturales y turistas: Unos leen lo que otros miran. Pasos. Revista de Turismo y Patrimonio Cultural, $1(1), 1-12$.

Sen, A. (1999). Development as freedom. New York: Alfred A. Knopf.

Sen, A. (2004). How does culture matter? En V. Rao \& M. Walton (Eds.). Culture and Public Action. Stanford: Stanford University Press.

Sen, A. (2006). Identity and Violence. London: Penguin Books.

Spence, R. (2009). Economic Growth. En S. Deneulin \& L. Shahani (Eds.). An introduction to the Human Development and Capability Approach. London: Earthscan.

Telfer, D. (2003). Development Issues in Destination Communities. En S. Singh, D. Timothy \& R. Dowling. Tourism in Destination Communities. Wallingford: CABI Publishing.

UNDP (1990). Human Development Report 1990. Concept and Measurement of human development. Chapter 1: Defining and measuring Human Development. Nueva York: United Nations Development Programme.

Urbano, H. (2012). La identidad en búsqueda de un patrimonio en los espacios turísticos. Cultura, 26, 179-189.

Urry, J. (2002a). Consuming places. New York: Taylor \& Francis e-Library.

Urry, J. (2002b). Mobility and connections. (Último acceso: 01/04/2013). Recuperado de www.ville-enmouvement.com/telechargement/ 040602/mobility.pdf 